\title{
A Network Pharmacology Approach to Uncover the Mechanisms of Shen-Qi-Di-Huang Decoction against Diabetic Nephropathy
}

\author{
Sha Di $\mathbb{D}^{1},{ }^{1}$ Lin Han $\mathbb{D}$, ${ }^{1}$ Qing Wang, ${ }^{1}$ Xinkui Liu, ${ }^{2}$ Yingying Yang $\mathbb{D}$, 1 \\ Fan Li $\left(\mathbb{0},{ }^{2}\right.$ Linhua Zhao $\left(\mathbb{D},{ }^{1}\right.$ and Xiaolin Tong $\mathbb{C}^{1,3}$ \\ ${ }^{1}$ Department of Endocrinology, Guanganmen Hospital, China Academy of Chinese Medical Sciences, Beijing 100054, China \\ ${ }^{2}$ Department of Clinical Chinese Pharmacy, School of Chinese Materia Medica, Beijing University of Chinese Medicine, \\ Beijing 100102, China \\ ${ }^{3}$ Shenzhen Hospital of Guangzhou University of Chinese Medicine, Shenzhen 518034, China
}

Correspondence should be addressed to Linhua Zhao; melonzhao@163.com and Xiaolin Tong; tongxiaolin@vip.163.com

Received 4 April 2018; Revised 15 September 2018; Accepted 11 October 2018; Published 1 November 2018

Academic Editor: Kuttulebbai N. S. Sirajudeen

Copyright (C) 2018 Sha Di et al. This is an open access article distributed under the Creative Commons Attribution License, which permits unrestricted use, distribution, and reproduction in any medium, provided the original work is properly cited.

\begin{abstract}
Shen-Qi-Di-Huang decoction (SQDHD), a well-known herbal formula from China, has been widely used in the treatment of diabetic nephropathy (DN). However, the pharmacological mechanisms of SQDHD have not been entirely elucidated. At first, we conducted a comprehensive literature search to identify the active constituents of SQDHD, determined their corresponding targets, and obtained known DN targets from several databases. A protein-protein interaction network was then built to explore the complex relations between SQDHD targets and those known to treat DN. Following the topological feature screening of each node in the network, 400 major targets of SQDHD were obtained. The pathway enrichment analysis results acquired from DAVID showed that the significant bioprocesses and pathways include oxidative stress, response to glucose, regulation of blood pressure, regulation of cell proliferation, cytokine-mediated signaling pathway, and the apoptotic signaling pathway. More interestingly, five key targets of SQDHD, named AKT1, AR, CTNNB1, EGFR, and ESR1, were significant in the regulation of the above bioprocesses and pathways. This study partially verified and predicted the pharmacological and molecular mechanisms of SQDHD on DN from a holistic perspective. This has laid the foundation for further experimental research and has expanded the rational application of SQDHD in clinical practice.
\end{abstract}

\section{Introduction}

Diabetic nephropathy (DN), a complex and multifaceted condition, is one of the main microvascular complications of diabetes mellitus, especially type 2 diabetes mellitus (T2DM) [1]. T2DM is an important cause of kidney failure, which presents the risk of development of hypertension. In 2010, $6.4 \%$ of the world's population was diagnosed with diabetes mellitus, and this value is expected to increase to $7.7 \%$ in 2030, in other words, from 285 million to 439 million adults [2]. DN is distinguished by the elevated albumin excretion rate and/or the transient increased glomerular filtration rate (GFR) [3]. The earliest sign of $\mathrm{DN}$ is microalbuminuria ( $>30 \mathrm{mg} /$ day), which develops into macroalbuminuria ( $>300$ $\mathrm{mg} /$ day) and decreased GFR, eventually leading to end-stagerenal disease (ESRD) $[4,5]$. The pathogenesis of DN has been associated with oxidative stress and inflammation caused by chronic high blood glucose [6-8], glucose metabolic disorder [9], hemodynamics, and hemorheology anomalies [10]. The current standard therapy includes intensive treatment and control of hyperglycemia and blood pressure. A blockade of the renin-angiotensin system (RAS) is also associated [11]; however, RAS combination therapy cannot prevent the progression of DN and is linked to an elevated rate of severe adverse events. Novel agents have shown controversial results or side effects [12] which makes it important to develop more efficient treatment to cure $\mathrm{DN}$ and reduce side effects.

Traditional Chinese Medicine (TCM) is widely propagated and used in more than 100 countries across the world owing to its satisfactory clinical efficacy [13]. SQDHD was documented in Shen Shi Zun Sheng Shu, which was written by Shen Jinao in 1773 during the Qing Dynasty. SQDHD 


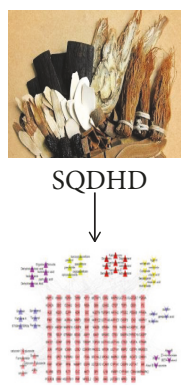

Compound-Compound Target Network

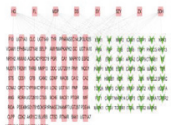

Herb-Compound Target-DN Target Network<smiles>[CH][CH]</smiles>

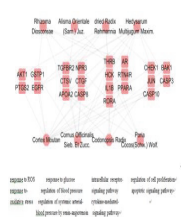

GO enrichment analysis

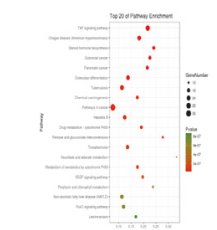

KEGG enrichment analysis

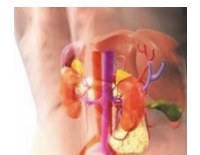

diabetic nephropathy

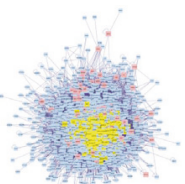

Compound Target-DN-Other Human Proteins' PPI Network

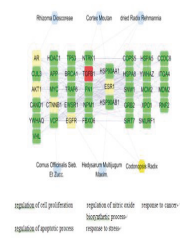

GO enrichment analysis

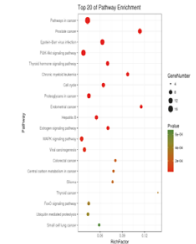

KEGG enrichment analysis

FIGURE 1: Workflow for SQDHD against diabetic nephropathy.

contains eight Chinese herbs, including Codonopsis Radix (Dang Shen [DS]), Hedysarum Multijugum Maxim. (Huang Qi [HQ]), dried Radix Rehmannia (Sheng Di Huang [SDH]), Rhizoma Dioscoreae (Shan Yao [SY]), Cornus Officinalis Sieb. Et Zucc. (Shan Zhu Yu [SZY]), Cortex Moutan (Mu Dan Pi [MDP]), Alisma Orientale (Sam.) Juz. (Ze Xie [ZX]), and Poria $\operatorname{Cocos}(S c h w$.) Wolf. (Fu Ling [FL]). Liuwei dihuang pill (LDP), including Cornus Officinalis Sieb. Et Zucc., Cortex Moutan, Rhizoma Dioscoreae, Poria Cocos(Schw.) Wolf., Alisma Orientale (Sam.) Juz., and Radix Rehmanniae Praeparata, inhibited erythrocyte aldose reductase activity and lowered urinary albumin excretion rate and beta2-MG in the blood and urine in the treated group compared to those in the control group [14]. LDP can decrease multiple pathways including TGF- $\beta /$ SMADS, MAPK, and NF- $\kappa \mathrm{B}$ signaling to prevent the progress of renal fibrosis and defend glomerular mesangial cells [15]. Astragaloside IV (ASI), active component in Hedysarum Multijugum Maxim., could inhibit high glucose-induced cell apoptosis and decrease TGF- $\beta 1$ and the activity of p38 in the MAPK pathway [16]. Dried Rehmanniae Radix reduced glucose, urea nitrogen, 5-hydroxymethylfurfural, and thiobarbituric acid- (TBA-) reactive substance levels in DN rats [17]. Moutan Cortex could significantly decrease blood glucose, serum creatinine, and urine protein in $\mathrm{DN}$ rats and reduce transforming growth factor beta 2 (TGF- $\beta 2$ ) in renal tissue [18]. Therefore, SQDHD might exhibit substantial effect on DN. As SQDHD includes many chemical compounds and adjusts a variety of targets, the pharmacological mechanisms require a complete clarification, which has been a challenge.

Network pharmacology, put forward by Hopkins in 2007, is used to elucidate the drugs effect on multiple targets [19]. Network pharmacology can build networks to reflect and clarify the interactive relationship between multiple components, multiple targets, multiple pathways, and complex diseases. It is also capable of interpreting the mechanisms of functional drugs based on the network built on public databases or available data through earlier researches. Network pharmacology can reconstruct a "drug target disease" network prediction model [20, 21]. TCM herbal formulas treat and prevent disease, and is composed of multicomponents and multitargets; thus, its mechanisms can be investigated by network pharmacology. The study was aimed at elucidating the pharmacological mechanisms of SQDHD in DN treatment using the comprehensive network pharmacology. The workflow of network pharmacologybased study of SQDHD against diabetic nephropathy was exhibited in Figure 1.

\section{Material and Methods}

\subsection{Data Preparation}

2.1.1. Composite Compounds of Each Herb in SQDHD. Seventy-seven active compounds from the eight herbs found in SQDHD were screened from domestic and foreign literatures, 
and the Traditional Chinese Medicine Systems Pharmacology Database [22] (TCMSP, http://lsp.nwu.edu.cn/tcmsp.php). TCMSP is a unique pharmacology platform for Chinese herbal medicines. Eleven compounds in Codonopsis Radix [23-29], 7 in Poria Cocos (Schw.) Wolf [30, 31], 11 in Hedysarum Multijugum Maxim [32-34], 9 in Cortex Moutan [35, 36], 12 in Rhizoma Dioscoreae [37-40], 12 in Cornus Officinalis Sieb. Et Zucc [41-51], 5 in Radix Rehmanniae $[52,53]$, and ten in Alisma Orientale (Sam.) Juz [54-56] were collected as well as the Canonical SMILES of all active compounds from TCMSP. The details are described in Table S1. Three-dimensional chemical structure data of active compounds was found and exported from PubChem [57] (https://pubchem.ncbi.nlm.nih.gov/).

2.1.2. Compound Targets for Each Herb in SQDHD. The compound targets of each herb found in SQDHD were collected from Stitch [58] (http://stitch.embl.de/, ver. 5.0), by inputting the Canonical SMILES into SMILES string(s), with the organism selected as "Homo sapiens" and a confidence score $>0.4$. The compound targets having no relationship with the compounds were deleted. Stitch is a resource to explore interactions between chemicals and proteins. Input 3D structure of active compounds into PharmMapper [59] (http://lilab.ecust.edu.cn/pharmmapper/index.php, Updated on Nov 27, 2017), a freely accessed web-server designed to identify potential target candidates for given probe small molecules (drugs, natural products, or other newly discovered compounds with binding targets unidentified) using the pharmacophore mapping approach. We used UniProtKB [60] (http://www.uniprot.org/) to obtain the standard compound targets' names. This database provides the scientific community with a comprehensive, high-quality, and freely accessible resource of protein sequence and functional information. The protein names were entered into UniProtKB, with the organism selected as "Homo sapiens," prior to the retrieval of the official symbol. We therefore obtained the compound targets for each herb in SQDHD. The details are described in Table S2.

2.1.3. DN Targets. The DN targets were obtained from the Therapeutic Target Database [61] (TTD, https://db.idrblab .org/ttd/, Updated in Sep. 10, 2017), Online Mendelian Inheritance in $\mathrm{Man}^{\circledR}$ [62] (OMIM, http://omim.org/about/), DrugBank [63] (https://www.drugbank.ca/, Updated in Dec. 20, 2017, ver. 5.0.11), and the Genetic Association Database (GAD, https://geneticassociationdb.nih.gov/). TTD provides information regarding the known and explored therapeutic protein and nucleic acid targets, the targeted disease, pathway information and the corresponding drugs directed at each of the targets. Seventeen targets for DN were collected and the details are described in Table S3. OMIM is a comprehensive, authoritative compendium of human genes and genetic phenotypes that is freely available and updated daily. The full-text, referenced overviews in OMIM contain information on all known mendelian disorders and over 15,000 genes. The DrugBank database is a comprehensive, freely accessible, online database containing information on drugs and drug targets. The GAD is a database of genetic associational data from complex diseases and disorders. After serving the scientific community for more than 10 years, GAD has been retired and all data is "frozen" as of 09/01/2014. However, all GAD data as of 08/18/2014 will continue to be available.

2.1.4. Protein-Protein Interaction Data. All protein-protein interaction (PPI) data were derived from STRING [64] (https://string-db.org/, ver. 10.5), and the organism was selected as "Homo sapiens" and a confidence score $>0.7$. The STRING database, an update on the online database, collects and presents known and predicted PPI with a confidence score and accessory information. The score represents the interaction confidence of the protein, which has a positive relationship [65].

\subsection{Network Construction}

2.2.1. Network Construction Method. The network construction was performed as follows: (1) the active compoundsactive compounds target network of SQDHD was built, (2) the herb-compound target-DN target network was built via linking the eight SQDHD herbs with compound targets of each herb, and DN targets, and (3) the compound targets-DN targets-other human proteins network was built.

We used the network visualization software Cytoscape [66] (http://cytoscape.org/, ver. 3.5.0) to build all networks. Cytoscape is an open source software platform for complex network analysis and visualization. It visualizes molecular interaction networks and biological pathways and integrating these networks with annotations, gene expression profiles, and other state data.

2.2.2. Network Topological Feature Set Definition. We used three indices to evaluate every node in the network.

The three indices contain degree, betweenness, and closeness of nodes. Degree represents the number of edges between a node and another node in the network [67]. Betweenness evaluates the participation of a node in the shortest parts of the network and reflects the ability of nodes to deal with the rate of information flow in the network as well [68]. Closeness is the inverse of the sum of the distance from a node to other nodes. The three indices play an important role in the network, and the level of the indices has a positive association with the importance of node in the network [69].

2.3. Gene Ontology Enrichment Analysis. The Database for Annotation, Visualization, and Integrated Discovery [70] (DAVID Bioinformatics Resources, https://david.ncifcrf.gov/, ver. 6.8) was utilized for the Gene Ontology (GO) enrichment analysis. DAVID provides a comprehensive set of functional annotation tools so that investigators can understand the biological meaning behind large lists of genes.

\section{Results and Discussion}

3.1. Compound-Compound Target Network Analysis. The network consists of 181 nodes (50 compounds in SQDHD and 131 compound targets) and 551 edges, as shown in Figure 2. The network suggests that many compound targets 


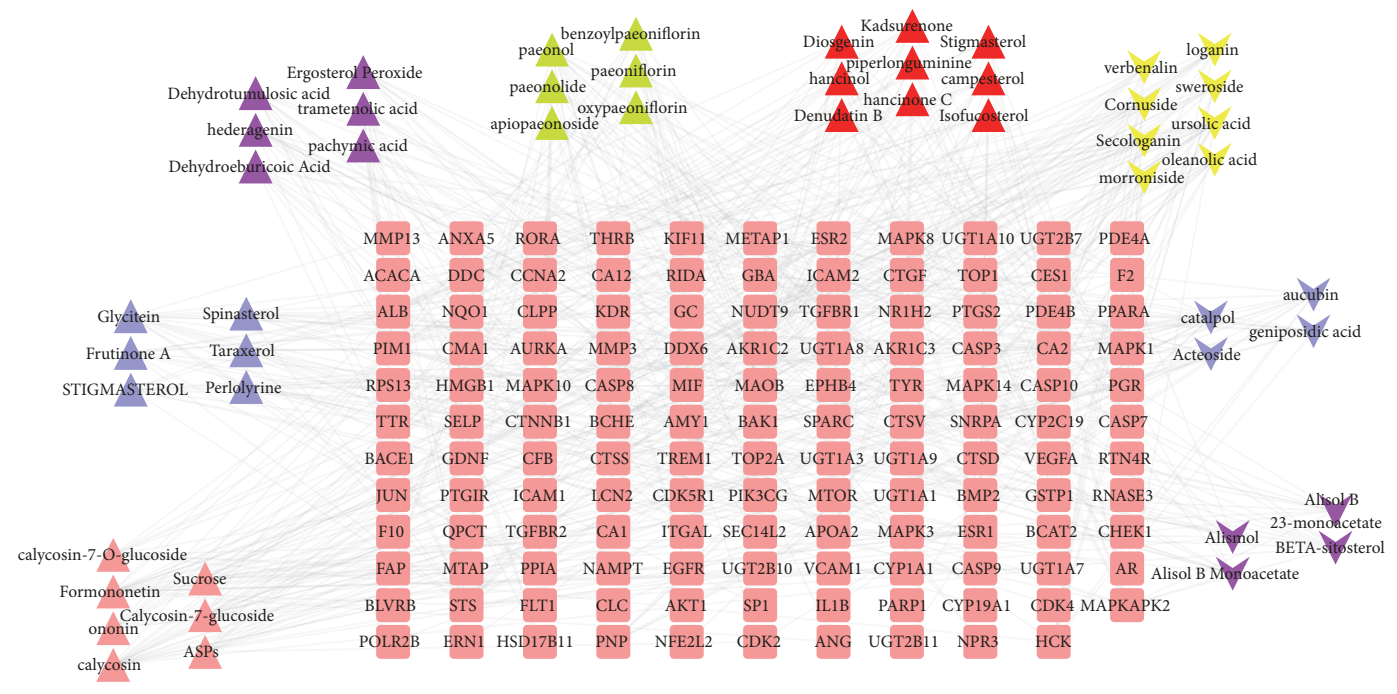

FIGURE 2: Compound-compound target network (pink rounded rectangles represent compound targets; pink triangles, blue triangles, purple triangles, green triangles, red triangles, yellow v, blue v, and purple v represent compounds of Hedysarum Multijugum Maxim., Codonopsis Radix, Poria Cocos(Schw.) Wolf., Cortex Moutan, Rhizoma Dioscoreae, Cornus Officinalis Sieb. Et Zucc., dried Radix Rehmannia, and Alisma Orientale (Sam.) Juz., respectively).

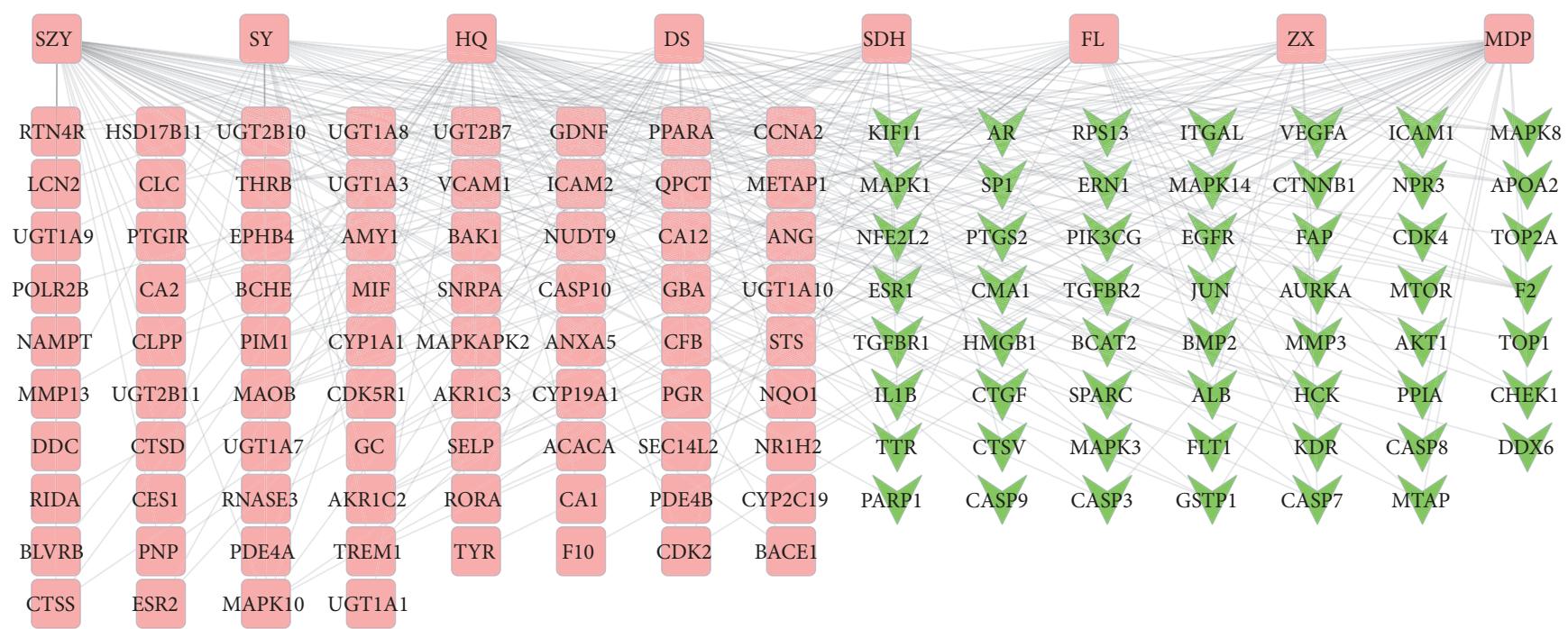

FiguRE 3: Herb-Compound Target-DN Target Network (the eight rounded rectangles at the top of the figure represent herbs, the ones below on the left (pink rounded rectangles) represent compound, and green $v$ represents compound targets/DN targets).

can be adjusted by multiple compounds, while the number of compound targets such as VEGF-A, CLC, AKT1, and PIK3CG can be regulated by just one compound. PIM1 can be regulated by 24 compounds, and STS, MAOB, CA2, and BMP2 can be regulated by 20 compounds, which may be vital compound targets in SQDHD. From the network, we can make a rough observation of the relationship between active compounds and compound targets.

3.2. Herb-Compound Target-DN Target Network Analysis. This network was built to show the relationship between eight herbs, compound targets, and DN targets. In Figure 3, the network consists of 139 nodes (eight herbs, 76 compound targets, and 55 compound targets/DN targets) and 300 edges. The network shows that the compound targets are also controlled by drug targets (DN targets), which suggests that drugs may indirectly regulate disease-related proteins, while SQDHD can directly affect these proteins. SQDHD may also indirectly affect drug targets by controlling related proteins (compound targets). We also found SZY (58) having the highest connection with other nodes, followed by HQ, MDF $(56,45)$. This suggests their significance in the network.

In Figure 4, according to the GO enrichment analysis, compound targets, $\mathrm{DN}$ targets, and compound targets/DN 


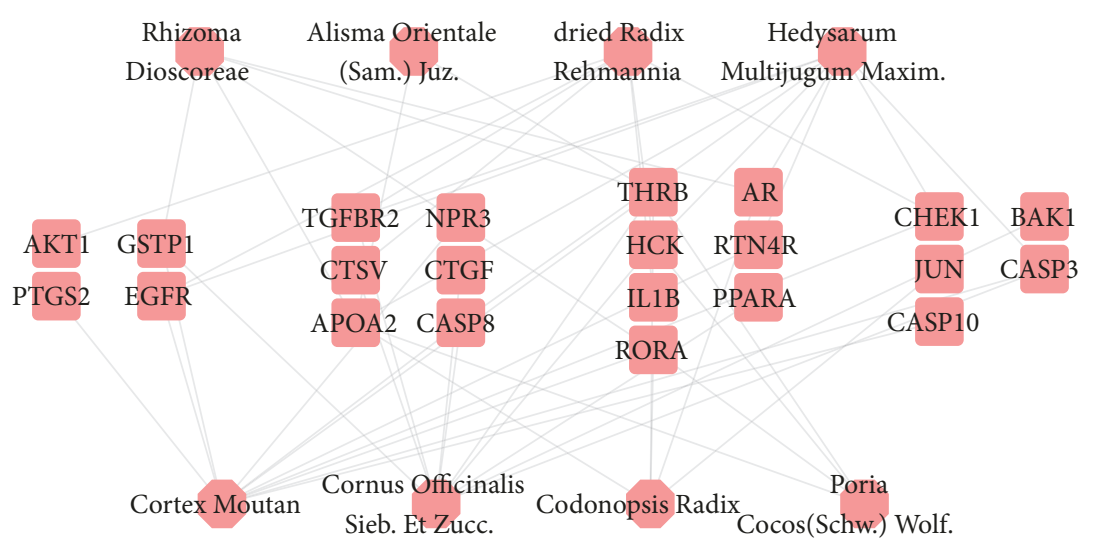

$\begin{array}{lccc}\text { response to ROS } & \text { response to glucose } & \text { intracellular receptor- } & \text { regulation of cell proliferation } \\ \text { response to- } & \text { regulation of blood pressure } & \text { signaling pathway } & \text { apoptotic signaling pathway } \\ \text { oxidative stress } & \text { regulation of systemic arterial- } & \text { cytokine-mediated- } & \\ & \text { blood pressure by renin-angiotensin } & \text { signaling pathway } & \end{array}$

FIGURE 4: According to the associated biological processes or pathways, compound targets of SQDHD and DN targets are related to various molecular mechanisms of DN (The pink octagons and pink rounded rectangles represent the eight herbs in SQDHD and targets, respectively).

targets are significantly associated with response to oxidative stress (GO:0006979; Fold Enrichment $=4.45 ; P=0.011$ ), response to reactive oxygen species (GO:0000302; Fold Enrichment $=8.36 ; P=0.011)$, response to glucose $(\mathrm{GO}$ : 0009749; Fold Enrichment $=10.79 ; P<0.001$ ), regulation of systemic arterial blood pressure by renin-angiotensin (GO:0003081; Fold Enrichment $=40.76 ; P=0.0021$ ), regulation of blood pressure (GO:0008217; Fold Enrichment = 13.79; $P<0.001$ ), regulation of cell proliferation (GO:0042127; Fold Enrichment $=4.41 ; P<0.001)$, cytokine-mediated signaling pathway (GO:0019221; Fold Enrichment $=4.36 ; P=$ 0.0054), apoptotic signaling pathway (GO:0097190; Fold Enrichment $=5.74 ; P=0.011)$, and intracellular receptor signaling pathway (GO:0030522; Fold Enrichment $=8.58 ; P$ $=0.011$ ). The details are described in Table S4.

In the network (Figure 4), there are numerous DNrelated biological processes, including response to oxidative stress (GO:0006979), response to glucose (GO: 0009749), regulation of blood pressure (GO:0008217), regulation of cell proliferation (GO:0042127), and cytokine-mediated signaling pathway (GO:0019221). These processes are the potential mechanisms involved in the treatment of DN. Some literature has reported several biological processes obtained using DAVID. Hypertension and hyperglycemia play vital roles in the processes of DN. Cytokines (vascular endothelial growth factor (VEGF), CC chemokine receptor 2 (CCR2), TGF- $\beta$, protein kinase $\mathrm{C}(\mathrm{PKC})$ ), oxidative stress, and inflammation are also key elements in the processes of DN. Currently, standard treatments for DN include controlling hyperglycemia and blood pressure by inhibiting RAS $[13,71,72]$. The RAS may increase renovascular resistance and intraglomerular pressure, leading to renal damage [73], hyperglycemia, rise in matrix production, or glycation of matrix proteins. These processes subsequently lead to hyperglycemia, which results in vessel injury followed by kidney mesangial expansion and injury [13].

Intracellular reactive oxygen species (ROS) increases the secretion by mitochondria in glucose stimulation. This occurs through five main pathways including the pathogenesis of complications: polyol pathway flux, increased formation of advanced glycation end products (AGEs), increased expression of the receptor for AGEs and its activating ligands, activation of PKC isoforms, and overactive hexosamine pathway. Elevated ROS leads to ischemia and defective angiogenesis and then activates many proinflammatory pathways [7476]. NO can negatively control mitochondrial oxidative metabolism, by binding to cytochrome c oxidase. ROS directly decreases eNOS activity [77]. The chronic hyperglycemic milieu markedly increases AGEs in both the cellular and extracellular compartments in various tissues [78, 79]. AGEs binding to the receptor for AGEs (RAGE) increase ROS production. ROS can regulate $\mathrm{PKC}$ pathway in mesangial cells and increase TGF- $\beta$ which is associated with kidney fibrosis. Meanwhile, ROS can also activate NF- $\kappa$ B in mesangial cells, leading to inflammatory response. ROS results in glomerular mesangial expansion and renal tubulointerstitial fibrosis, via disturbing cell function, and signal transduction cascades $[9,80,81]$. Insulin-dependent diabetic BB rats and NOD mouse had elevated expression of TGF- $\beta$ in the kidney, and plasma and urine of DN patients had higher VEGF. This suggests the urinary VEGF might be used as a sensitive marker of DN and for predicting disease progression [82, 83].

In Figure 5, according to KEGG enrichment analysis, compound targets, DN targets, and compound targets/DN 


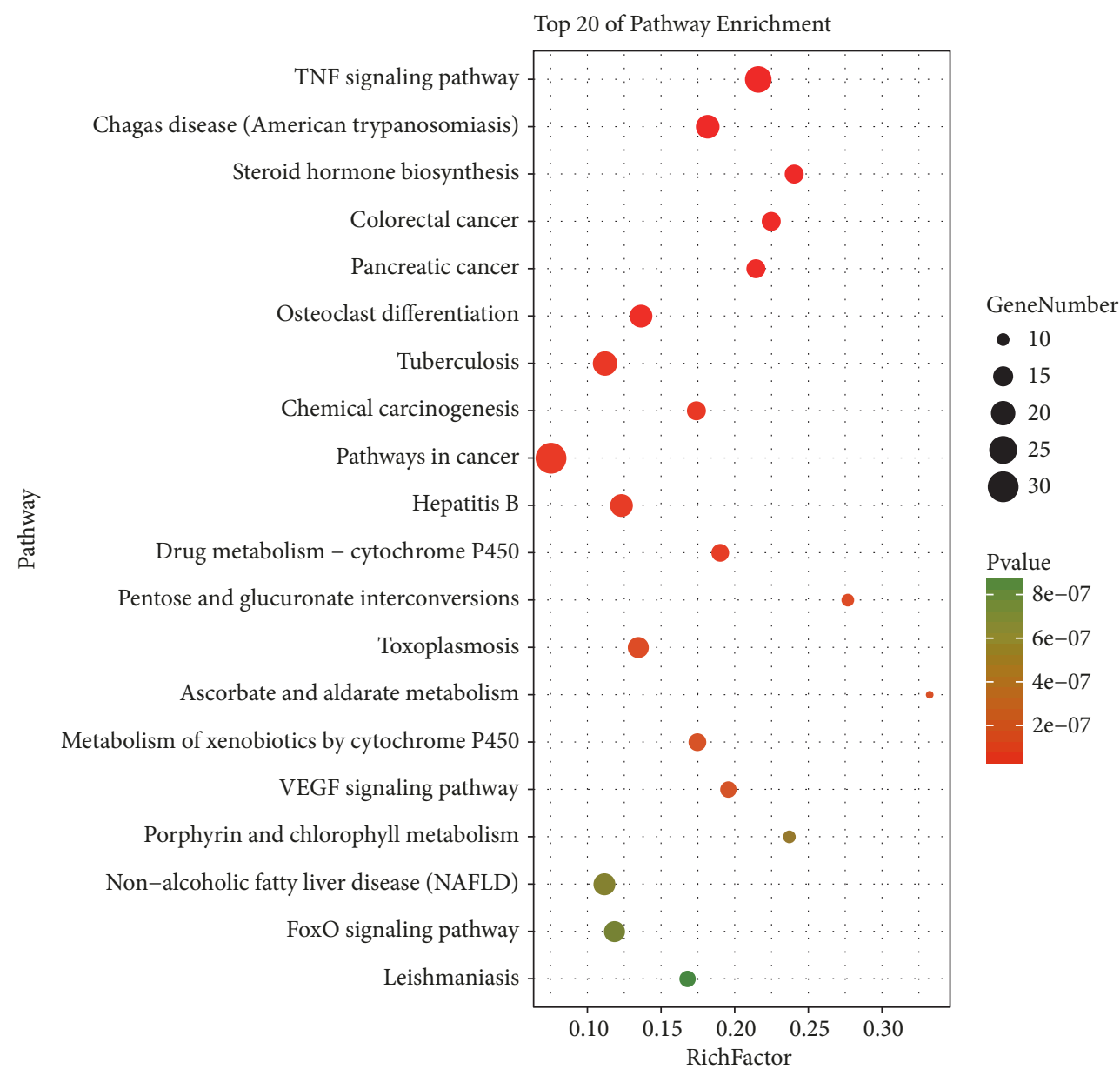

FIGURE 5: Differentially expressed gene PATHWAY enrichment point diagram (the vertical axis represents the pathway name, the horizontal axis represents the Rich factor, the size of the dot indicates the number of genes expressed in the pathway, and the color of the dot corresponds to the different Qvalue range).

targets are significantly associated with TNF signaling pathway, Chagas disease, steroid hormone biosynthesis, tuberculosis, VEGF signaling pathway and others. The most enriched pathways involving significant differential expression are TNF signaling pathway [84], steroid hormone biosynthesis $[85,86]$, colorectal cancer [87], pancreatic cancer, glucuronate interconversions [88], and VEGF signaling pathway [89]. The details are described in Table S5.

3.3. Compound Target-DN-Other Human Proteins' PPI Network Analysis. We built the compound target-DN targetother human proteins' PPI network to evaluate the significance of compound targets. The network has 1626 nodes (123 compound targets, 82 DN targets, 8 compound-DN targets, and 1413 other human proteins which have a connection with compound targets or DN targets) and 36768 edges (Figure 6). The significant targets are 400 nodes, which have 13,722 edges evaluated by the three indices including degree, betweenness, and closeness (degree $\geq 60$, betweenness $\geq 270.71207$, and closeness $\geq 0.41004288$ ). Finally, we selected 40 main nodes, which play an important role in the network (Figure 7). The details are described in Table S6.
Furthermore, the significant compound Target-DNOther Human Proteins' PPI Network has 400 major nodes and 13,722 edges, with the 40 main nodes include five compound targets, 1 compound target and/or DN target, and 34 other human proteins interacting with the compound or DN targets. In Figure 8, according to GO enrichment analysis of the 40 main targets, a significant connection with a negative regulation of the apoptotic process (GO: 0043066; Fold Enrichment $=8.74 ; P<0.001)$, regulation of signal transduction by p53 class mediator (GO: 1901796; Fold Enrichment $=23.69 ; P<0.001)$, positive regulation of nitric oxide biosynthetic process (GO: 0045429; Fold Enrichment = $48.81 ; P<0.001$ ), positive regulation of cell proliferation (GO: 0008284; Fold Enrichment $=8.10 ; P<0.001)$, response to stress (GO: 0006950; Fold Enrichment $=27.52 ; P<0.001$ ), a negative regulation of the TGF- $\beta$ receptor signaling pathway (GO: 0030512; Fold Enrichment $=26.23 ; P<0.001)$, and regulation of nitric oxide synthase activity (GO: 0050999; Fold Enrichment $=48.43 ; P=0.0016)$. The details are described in Table S7.

In Figure 9, according to KEGG enrichment analysis, compound targets, DN targets, and other human proteins are 


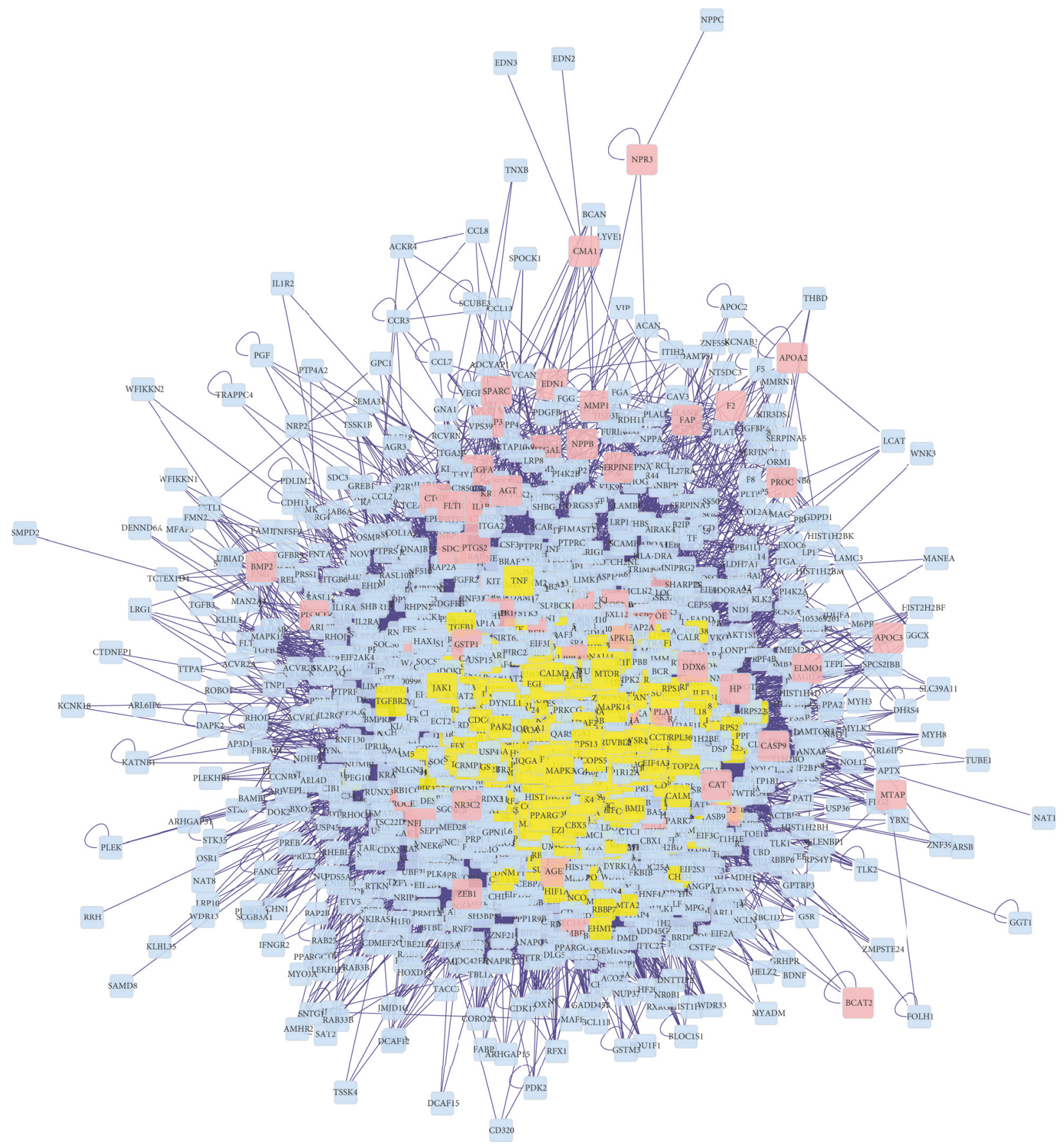

FIgure 6: Compound Target-DN-Other Human Proteins' PPI Network (pink nodes, yellow nodes, blue nodes represent compound targets and DN targets, the significant targets, and other human proteins interacting with the compound or DN targets, respectively).

significantly associated with the PI3K-Akt signaling pathway, cell cycle, MAPK signaling pathway, and others. The details are described in Table S8.

The largest number of targets, biological processes, and pathways related to SQDHD and DN has been reported in some literature. Nitric oxide (NO) is one such example that has actively been associated with the kidneys through several segments consisting of the regulation of renal hemodynamics, renin secretion, inhibition of tubular sodium reabsorption, tubuloglomerular feedback (TGF), and renal sympathetic nerve activity [90, 91]. NO synthase (NOS), including neuronal NOS (nNOS or NOS1), inducible NOS (iNOS or NOS2), and endothelial NOS (eNOS or NOS3), can promote the synthesis of NO that dilates blood vessels. NOS inhibitor (L-NAME) was used for Otsuka LongEvans Tokushima Fatty spontaneous diabetic rat models and Long-Evans Tokushima Otuska rat models as age-matched controls. As a result, the diabetic group had lower urinary 

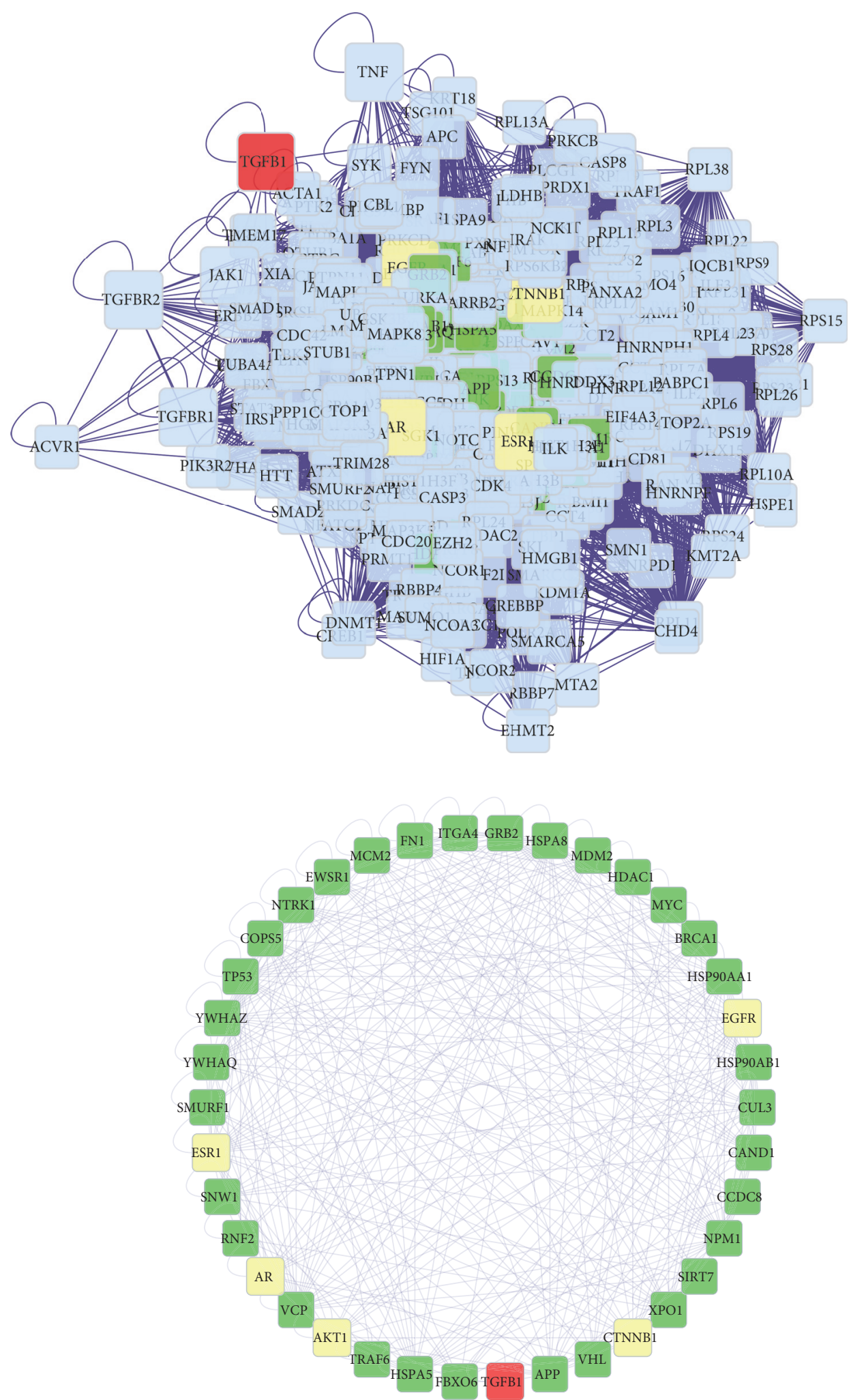

FIgURE 7: The significant compound Target-DN-Other Human Proteins' PPI Network (degree $\geqslant 60$, betweenness $\geqslant 270.71207$, and closeness $\geqslant 0.41004288$; yellow nodes, red nodes, green nodes, and blue nodes represent compound targets, compound targets/DN targets, and other human proteins interacting with the compound or DN targets, respectively). 


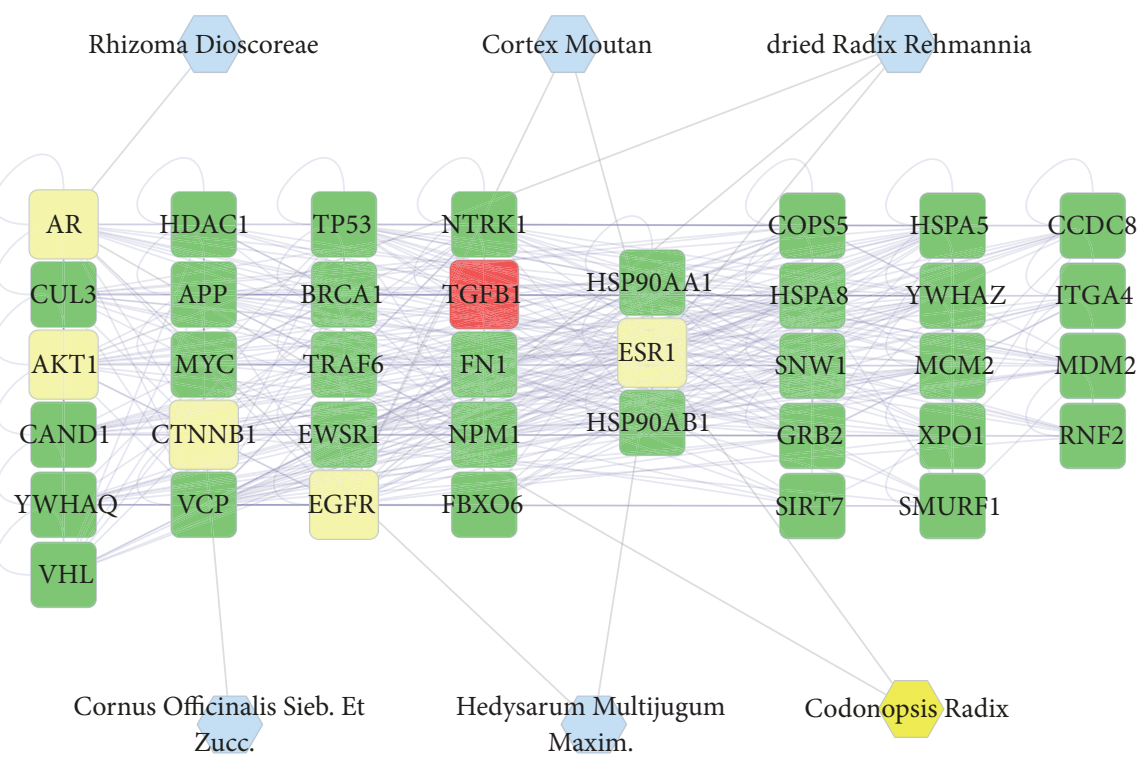

regulation of cell proliferation

regulation of nitric oxide

response to cancer

biosynthetic process

regulation of apoptotic process

response to stress

FIGURE 8: Direct interaction network between 40 major nodes in the SQDHD compound target-DN target-other human proteins' PPI network. According to the associated biological processes, compound targets of SQDHD and DN targets are related to various molecular mechanisms of DN (yellow nodes, red nodes, green nodes, and blue nodes represent compound targets, compound targets/DN targets, and other human proteins interacting with compound targets or DN targets respectively).

$\mathrm{NO} 2+\mathrm{NO} 3$ and higher urinary protein compared to the control groups. The NOS inhibitor was observed to aggravate the diabetic kidney disease [92]. Research has reported that posttranslational regulation and phosphorylation of nNOS and eNOS play vital roles in activating and inhibiting $\mathrm{NO}$ synthesis in the kidney [91]. Sedentary Zucker diabetic fatty (Sed-ZDF) rats had lower eNOS and nNOS expression than male ZDF rats with exercise for 8 weeks [93].

Chronic elevated blood glucose level in diabetics results in oxidative stress and inflammation associated with diabetic complications such as DN. ROS/NS overproduction caused by elevated glucose levels and enzymatic and nonenzymatic antioxidant defense deficiency can lead to oxidative stress. The influence of ROS/NS in cell signaling pathways has been linked to tissue metabolism, cell proliferation, and cell death [94]. The markers for ROS/NS damage is also seen to rise in diabetic kidneys. Research has reported that TGF- $\beta$ in the diabetic kidney cortex is related to oxidative stress, vascular cell adhesion molecule 1 (VCAM-1), and monocyte chemotactic protein 1 (MCP-1) [9].

Several growth factors and cytokines, including TGF- $\beta$, VEGF, PDGF, CTGF, and others, are likely mediators of the influence of high blood glucose level on the kidneys. TGF$\beta 1$ has been proven as the vital cytokine that is linked to the glomerular pathology of the extracellular matrix (ECM) that is typically seen in DN. TGF- $\beta 1$ prevents cell proliferation and apoptosis, although inducing hyperplasia and hypertrophy of mesangial cells. Diabetic animals had increased TGF- $\beta$ in the glomerular [95]. TGF- $\beta 1$-dependent and protein kinase $C$ dependent pathways induce CTGF in high glucose, and CTGF may be a mediator in the process of matrix production driven by TGF- $\beta 1$ [96]. VEGF-A, a family of secreted glycoprotein isoforms, is an endogenous protective factor and mainly produced by podocytes in glomeruli. Elevated VEGFA protects the glomerular microvasculature in diabetics, prevents apoptosis of vascular wall cells, and prevents the development of DN $[97,98]$. Targets such as FBXO6, HSPAS, HSPA5, SIRTT, and the pathway involved have shown that the SQDHD compound targets, DN target, and other human proteins have great relationship in cancer regulation, though the reported research lacks vital information. Therefore, the role of SQDHD in the regulation of cancer should be further studied.

Intensive treatment of hyperglycemia and hypertension is the primary treatment for DN. Western medicines, including $\mathrm{ACEI}$ and $\mathrm{ARB}$, can protect renal function of $\mathrm{DN}$; owing to shortage, a sufficient amount is lacking to delay or retard the progression of DN. TCM has been used in treating diabetes and preventing its complications. Hachimijiogan (HJG), consisting of Rehmannia radix, Fructus Corni, and others, could decrease TGF- $\beta 1$ and iNOS levels in the kidney cortex and reduce urinary protein, serum glycosylated protein and AGEs. LDP, including Rehmannia glutinosa, Fructus Corni, cortex Mountain, Dioscorea opposita, Poria Cocos, and Alisma Orientale, decreases urinary albumin excretion rate levels. Astragalus (Huang Qi in Chinese) could prevent the early 


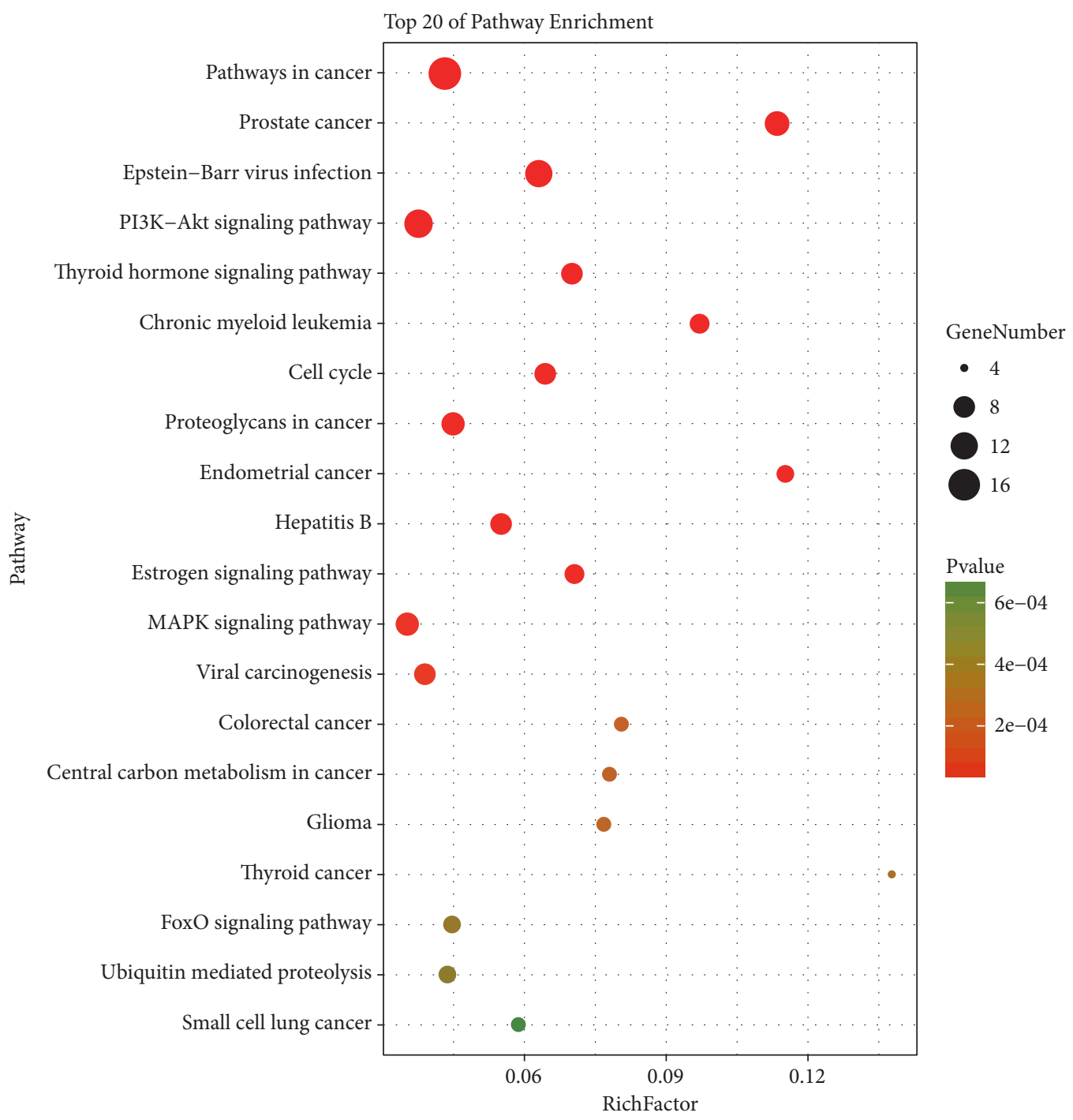

FIGURE 9: Differentially expressed gene PATHWAY enrichment point diagram of compound Target-DN-Other Human targets.

proliferation of mesangial cell, AGEs-mediated cell apoptosis, and reduce TGF- $\beta 1$ expression. Moutan cortex can ameliorate inflammation via targeting RAGE in vitro or in vivo. Rehmannia Radix (Di Huang) can reduce TGF- $\beta 1$, CTGF, and Ang II in high fat diet-fed plus STZ-induced diabetic rats [99, 100]. In Figure 8, according to KEGG enrichment analysis, compound target-DN target-other human proteins are significantly related to pathways in cancer, the estrogen signaling pathway, cell cycle, and, as previously mentioned, the PI3KAKT signaling pathway, thyroid hormone signaling pathway, and MAPK signaling pathway. PI3K-AKT signaling pathway has been indicated as the source of glomerular hypertrophy and ECM accumulation [101, 102]. Some research reported that the thyroid hormone, triiodothyronine (T3), can inhibit transcriptional activation of TGF- $\beta / \mathrm{SMAD}$ via binding to its nuclear receptors (TRs) [103]. A reduction in the activity of the Akt signaling pathway, which is controlled by hyperglycemia, may activate p38 MAPK, inflammatory and fibrotic markers, further leading to DN [104]. P38-MAPK in vascular smooth muscle cells and aorta can be phosphorylated by hyperglycemia in diabetic rats [105].

Therefore, previous research as well as our study has shown that SQDHD can treat and prevent diabetes and its complication, including $\mathrm{DN}$, via regulating several targets linked to the NO biosynthetic process, nitric oxide synthase activity, cell apoptosis, cell proliferation, and stress. Some mechanisms of SQDHD on DN covered by network pharmacology have been reported, and new mechanisms can be further verified with animal and cell experiments.

\section{Conclusion}

The TCM herbal formula, a vital type of complementary and alternative medicine, is widely used to treat diabetes mellitus and complications. The study shows that SQDHD may attenuate high glucose level, high blood pressure, oxidative stress, cell apoptosis, and proliferation related to $\mathrm{DN}$, through the adjustment of its candidate targets and 
pathway by employing network pharmacology. Uncovering the pharmacological mechanism and demonstrating these prediction targets with experiments are required in future studies.

\section{Data Availability}

The data used to support the findings of this study are available from the corresponding author upon request.

\section{Conflicts of Interest}

The authors declared that they have no conflicts of interest.

\section{Authors' Contributions}

Dr. Sha Di and Lin Han contributed equally to this work.

\section{Acknowledgments}

This work was financially supported by the National Natural Science Foundation of China (no. 81430097) and China Academy of Chinese Medical Sciences (no. ZZ0808004).

\section{Supplementary Materials}

Table S1: Composite compounds of each herb in SQDHD. Table S2: Compound targets for each herb in SQDHD. Table S3: DN targets. Table S4: Enrichment analysis of compound target, DN target, and compound target/DN target based on Gene Ontology (GO) annotation. Table S5: Enrichment analysis of compound target, DN target, compound target/DN target, and other human protein based on Gene Ontology (GO) annotation. Table S6: Topological features of major nodes. Table S7: Enrichment analysis of compound targetDN-other human proteins based on Gene ontology (GO) annotation. (Supplementary Materials)

\section{References}

[1] G. B. Piccoli, G. Grassi, G. Cabiddu et al., "Diabetic Kidney Disease: A Syndrome Rather Than a Single Disease," The Review of Diabetic Studies, vol. 12, no. 1-2, pp. 87-109, 2015.

[2] J. E. Shaw, R. A. Sicree, and P. Z. Zimmet, "Global estimates of the prevalence of diabetes for 2010 and 2030," Diabetes Research and Clinical Practice, vol. 87, no. 1, pp. 4-14, 2010.

[3] G. Jerums, E. Premaratne, S. Panagiotopoulos, S. Clarke, D. A. Power, and R. J. MacIsaac, "New and old markers of progression of diabetic nephropathy," Diabetes Research and Clinical Practice, vol. 82, no. 1, pp. S30-S37, 2008.

[4] M. V. Rocco and J. S. Berns, "KDOQI in the Era of Global Guidelines," American Journal of Kidney Diseases, vol. 54, no. 5, pp. 781-787, 2009.

[5] K. Reidy, H. M. Kang, T. Hostetter, and K. Susztak, "Molecular mechanisms of diabetic kidney disease," The Journal of Clinical Investigation, vol. 124, no. 6, pp. 2333-2340, 2014.

[6] R. Lindblom, G. Higgins, M. Coughlan, and J. B. de Haan, "Targeting mitochondria and reactive oxygen species-driven pathogenesis in diabetic nephropathy," Review of Diabetic Studies, vol. 12, no. 1-2, pp. 134-156, 2015.
[7] P. B. Pal, K. Sinha, and P. C. Sil, "Mangiferin attenuates diabetic nephropathy by inhibiting oxidative stress mediated signaling cascade, TNF $\alpha$ related and mitochondrial dependent apoptotic pathways in streptozotocin-induced diabetic rats," PLOS ONE, vol. 9, no. 9, Article ID e107220, 2014.

[8] R. Mohamed, C. Jayakumar, F. Chen et al., "Low-dose IL-17 therapy prevents and reverses diabetic nephropathy, metabolic syndrome, and associated organ fibrosis," Journal of the American Society of Nephrology, vol. 27, no. 3, pp. 745-765, 2016.

[9] R. Mishra, S. N. Emancipator, T. Kern, and M. S. Simonson, "High glucose evokes an intrinsic proapoptotic signaling pathway in mesangial cells," Kidney International, vol. 67, no. 1, pp. 82-93, 2005.

[10] F. Gregorio, F. Ambrosi, F. Carle et al., "Microalbuminuria, brain vasomotor reactivity, carotid and kidney arterial flow in Type 2 diabetes mellitus.," Diabetes, nutrition \& metabolism, vol. 17, no. 6, pp. 323-330, 2004.

[11] Y. Pan, Y. Huang, Z. Wang et al., "Inhibition of MAPK-mediated ACE expression by compound C66 prevents STZ-induced diabetic nephropathy," Journal of Cellular and Molecular Medicine, vol. 18, no. 2, pp. 231-241, 2014.

[12] E. Dounousi, A. Duni, K. Leivaditis, V. Vaios, T. Eleftheriadis, and V. Liakopoulos, "Improvements in the management of diabetic nephropathy," Review of Diabetic Studies, vol. 12, no. 1-2, pp. 119-133, 2015.

[13] J. Wang, Y. Guo, and G. L. Li, "Current status of standardization of Traditional Chinese Medicine in China," Evidence-Based Complementary and Alternative Medicine, vol. 2016, Article ID 9123103, 7 pages, 2016.

[14] X. Y. Song, Q. Chen, and X. Y. Qi, "Effect of liuwei dihuang pill on erythrocyte aldose reductase activity in early diabetic nephropathy patients," Chinese Journal of Integrated Traditional and Western Medicine, vol. 24, no. 12, pp. 1087-1090, 2004.

[15] Z. J. Xu, S. Shu, Z. J. Li, Y. M. Liu, R. Y. Zhang, and Y. Zhang, "Liuwei Dihuang pill treats diabetic nephropathy in rats by inhibiting of TGF- $\beta /$ SMADS, MAPK, and NF-kB and upregulating expression of cytoglobin in renal tissues," Medicine, vol. 96, no. 3, p. e5879, 2017.

[16] Q. Wang, X. Shao, W. Xu et al., "Astragalosides IV inhibits high glucose-induced cell apoptosis through HGF activation in cultured human tubular epithelial cells," Renal Failure, vol. 36, no. 3, pp. 400-406, 2014.

[17] T. Yokozawa, H. Y. Kim, and N. Yamabe, "Amelioration of diabetic nephropathy by dried Rehmanniae Radix (Di Huang) extract," American Journal of Chinese Medicine, vol. 32, no. 6, pp. 829-839, 2004.

[18] M. Zhang, L. Feng, J. Gu et al., "The attenuation of Moutan cortex on oxidative stress for renal injury in AGEs-induced mesangial cell dysfunction and streptozotocin-induced diabetic nephropathy rats," Oxidative Medicine and Cellular Longevity, vol. 2014, Article ID 463815, 13 pages, 2014.

[19] A. L. Hopkins, "Network pharmacology," Nature Biotechnology, vol. 25, no. 10, pp. 1110-1111, 2007.

[20] G.-b. Zhang, Q.-y. Li, Q.-1. Chen, and S.-b. Su, "Network pharmacology: a new approach for chinese herbal medicine research," Evidence-Based Complementary and Alternative Medicine, vol. 2013, Article ID 621423, 9 pages, 2013.

[21] S. Li, T.-P. Fan, W. Jia, A. Lu, and W. Zhang, "Network pharmacology in Traditional Chinese Medicine," Evidence-Based Complementary and Alternative Medicine, vol. 2014, Article ID 138460, 2 pages, 2014. 
[22] J. Ru, P. Li, J. Wang et al., "TCMSP: a database of systems pharmacology for drug discovery from herbal medicines," Journal of Cheminformatics, vol. 6, no. 1, article 13, 2014.

[23] L.-j. Guan, Determination of the Ingredients of Codonopsis Radix from Different Sources and Study on the HPLC Specific Chromatograms of Codonopsis Radix, Shanxi Medical University, Taiyuan, China, 2015.

[24] Y.-j. Feng, P.-y. Zhuang, D.-y. Zhang, L. Gao, J.-m. Chen, and G. Han, "Study on chemical constituents of Codonopsis pilosula," China Journal of Chinese Materia Medica, vol. 1, 2017.

[25] W.-s. Liu, Study on The Extraction of The Codonopsis Pilosula Polysaccharide and The Codonopsis Pilosula Saponin and The Effects on Immune Ability and Anti-Oxidation for Mouse, Shanxi Agricultural University, Jinzhong, China, 2004.

[26] R. Zhu, Analyses and Anti-Tumor Activities of The Polysaccharides From Codonopsis pillosula, Northeast Normal University, Changchun, China, 2013.

[27] X.-X. Wang, P.-Y. Zhuang, J.-M. Chen, Y.-K. Yang, X.-Y. Lin, and D.-Y. Zhang, "Study on chemical constituents of Codonopsis pilosula," Chinese Traditional and Herbal Drugs, vol. 48, no. 9, pp. 1719-1723, 2017.

[28] J.-j. Zhang and C.-1. Hu, "Advances of modern in studies on Codonopsis Pilosula," Journal of Gansu Normal Colleges, vol. 22, no. 3, 2017.

[29] J.-Y. He, N. Ma, S. Zhu, K. Komatsu, Z.-Y. Li, and W.-M. $\mathrm{Fu}$, "The genus Codonopsis (Campanulaceae): A review of phytochemistry, bioactivity and quality control," Journal of Natural Medicines, vol. 69, no. 1, pp. 1-21, 2015.

[30] K-f. Wang, Study on Chemical Composition And Quality Control of Poria cocos, Beijing University of Chinese Medicine, Beijing, China, 2014.

[31] C. L. Chang, T. H. Li, C. L. Hou, and W. C. Yang, "Antihyperglycemic properties of crude extract and triterpenes from poria cocos," Evidence-Based Complementary and Alternative Medicine, vol. 2011, Article ID 128402, 8 pages, 2011.

[32] M. H. Liu, P. Li, X. Zeng, H. X. Wu, W. W. Su, and J. Y. $\mathrm{He}$, "Identification and pharmacokinetics of multiple potential bioactive constituents after oral administration of Radix Astragali on cyclophosphamide-induced immunosuppression in Balb/c mice," International Journal of Molecular Sciences, vol. 16, no. 3, pp. 5047-5071, 2015.

[33] Y.-C. Juan, Y.-H. Kuo, C.-C. Chang et al., "Administration of a Decoction of Sucrose- and Polysaccharide-Rich Radix Astragali (Huang Qi) Ameliorated Insulin Resistance and Fatty Liver but Affected Beta-Cell Function in Type 2 Diabetic Rats," Evidence-Based Complementary and Alternative Medicine, vol. 2011, Article ID 349807, 11 pages, 2011.

[34] R. L. C. Hoo, J. Y. L. Wong, C. F. Qiao, A. Xu, H. X. Xu, and K. S. L. Lam, "The effective fraction isolated from Radix Astragali alleviates glucose intolerance, insulin resistance and hypertriglyceridemia in $\mathrm{db} / \mathrm{db}$ diabetic mice through its antiinflammatory activity," Journal of Nutrition and Metabolism, vol. 7, article 67, 2010.

[35] Y. Wang, "Summary on pharmacological and analysing methods of the effective ingredients of cortex moutan," Asia-Pacific Traditional Medicine, vol. 12, no. 16, 2016.

[36] Y. Yuan, J. Yu, C. Jiang et al., "Functional diversity of genes for the biosynthesis of paeoniflorin and its derivatives in Paeonia," International Journal of Molecular Sciences, vol. 14, no. 9, pp. 18502-18519, 2013.
[37] F.-1. He, Extraction Active Components in Dioscoreae Rhizome and Research on Hypoglycemic Bioactive, Southwest University, Chongqing, China, 2011.

[38] F.-1. He, X.-l. Ye, X.-g. Li, and X.-f. Li, "Study on filtration and comparison of hypoglycemic bioactive components in dioscoreae rhizome," Science and Technology of Food Industry, vol. 32, no. 6, pp. 373-375, 2011.

[39] C. Guo, G. Ding, W. Huang, Z. Wang, Z. Meng, and W. Xiao, "Total saponin of Dioscoreae hypoglaucae rhizoma ameliorates streptozotocin-induced diabetic nephropathy," Drug Design, Development and Therapy, vol. 10, pp. 799-810, 2016.

[40] M. Jesus, A. P. J. Martins, E. Gallardo, and S. Silvestre, "Diosgenin: recent highlights on pharmacology and analytical methodology," Journal of Analytical Methods in Chemistry, vol. 2016, Article ID 4156293, 16 pages, 2016.

[41] J.-r. Liang, Study on Chemical Constituents and Bioactivity of Cornus officinalis, Northwest University, 2014.

[42] X.-s. Ye, J.-1. Zhang, and et al, "Chemical constituents from ripe fruit of Cornus officinalis," China Journal of Chinese Materia Medica, vol. 41, no. 24, pp. 4605-4609, 2016.

[43] L.-t. Zhang, Y.-f. Du, and et al, "Recent development and prospect of Cornus officinalis," China Journal of Chinese Materia Medica, vol. 35, no. 8, pp. 952-955, 2004.

[44] J. Yang, C.-r. Ji, and et al, "Identification of chemical constituents of Cornus officinalis," Chinese Traditional and Herbal Drugs, vol. 36, no. 12, pp. 1780-1782, 2005.

[45] J.-z. Dai and T. Lei, "Research progress on chemical constituents and pharmacological activities of Cornus officinalis," Journal of Chinese Pharmaceutical Sciences, vol. 15, no. 2, pp. 74-75, 2006.

[46] W.-x. Pi, H.-q. Xu et al., "The effect of the Iridoid Glycosides from Fructus Corni on serum SOD of Diabetic vascular complications in rats," Traditional Chinese Drug Research And Clinical Pharmacology, vol. 14, no. 1, pp. 23-24, 2003.

[47] H. Liu, "Advancement in research of fructus Corni Officinalis and its main components," Journal of Nanjing University of Traditional Chinese Medicine, vol. 19, no. 4, pp. 254-256, 2003.

[48] X.-n. Li, Studies on Isolation and Pharmacokinetics of Chemical Constituents from Fructus Corni, Hebei Medical University, 2007.

[49] Y. Pan, "Summary of the sudies of the chemical composition of Dogwood Fruit," Journal of Nanjing University of Traditional Chinese Medicine, no. 1, pp. 61-62, 1998.

[50] Q. Song, "Research progress of pharmacological activities of Fructus Corni," Information on Traditional Chinese Medicine, vol. 23, no. 2, pp. 24-25, 2006.

[51] G. Cao, Y. Zhang, X. D. Cong et al., "Research progress on the chemical constituents and pharmacological activities of Fructus corni," Journal of Chinese Pharmaceutical Sciences, vol. 18, 2009.

[52] W.-x. Liu, Studies on Extraction, Separation, Determination and Hypoglycemic Activity of Effective Components in Fresh Rehmannia Root, Academy of Military Medical Sciences, 2009.

[53] W.-x. Liu, H.-t. Du, and Z.-z. Wu, "Pharmacological actions of Radix Rehmanniae and its active components: research advances," Journal of International Pharmaceutical Research, vol. 36, no. 4, pp. 277-280, 2009.

[54] Z.-z. Xing and Z. Yu, "Research progress on chemical constituents and pharmacological effects of zexie (Alisma orientalis)," Guiding Journal of Traditional Chinese Medicine and Pharmacology, no. 15, pp. 75-78, 2017.

[55] T. Tian, Y.-l. Feng et al., "Research Progress on pharmacological and toxicological effects of Alisma orientalis," Journal of Chinese Medicinal Materials, vol. 37, no. 11, pp. 2103-2108, 2014. 
[56] J. J. Yoon, Y. J. Lee, S. M. Lee, D. G. Kang, and H. S. Lee, "Oryeongsan suppressed high glucose-induced mesangial fibrosis," BMC Complementary and Alternative Medicine, vol. 15, no. 1, article 30, 2015.

[57] W. Ihlenfeldt, "A virtual file system for the PubChem chemical structure and bioassay database," Chemistry Central Journal, vol. 2, Supplement 1, article P26, 2008.

[58] M. Kuhn, D. Szklarczyk, S. Pletscher-Frankild et al., "STITCH 4: integration of protein-chemical interactions with user data," Nucleic Acids Research, vol. 42, no. 1, pp. D401-D407, 2014.

[59] X. Wang, Y. Shen, S. Wang et al., "PharmMapper 2017 update: A web server for potential drug target identification with a comprehensive target pharmacophore database," Nucleic Acids Research, vol. 45, no. 1, pp. W356-W360, 2017.

[60] L. Breuza, S. Poux, A. Estreicher et al., "The UniProtKB guide to the human proteome," Database, vol. 2016, 2016.

[61] Y. H. Li, C. Y. Yu, X. X. Li et al., "Therapeutic target database update 2018: enriched resource for facilitating bench-to-clinic research of targeted therapeutics," Nucleic Acids Research, 2017.

[62] J. Amberger, C. A. Bocchini, A. F. Scott, and A. Hamosh, "McKusick's Online Mendelian Inheritance in Man (OMIM)," Nucleic Acids Research, vol. 37, no. 1, pp. D793-D796, 2009.

[63] D. S. Wishart, Y. D. Feunang, A. C. Guo et al., "DrugBank 5.0: A major update to the DrugBank database for 2018," Nucleic Acids Research, vol. 46, no. 1, pp. D1074-D1082, 2018.

[64] S. Hillenmeyer, L. K. Davis, E. R. Gamazon, E. H. Cook, N. J. Cox, and R. B. Altman, "STAMS: STRING-assisted module search for genome wide association studies and application to autism," Bioinformatics, vol. 32, no. 24, pp. 3815-3822, 2016.

[65] D. Szklarczyk, A. Franceschini, M. Kuhn et al., "The STRING database in 2011: functional interaction networks of proteins, globally integrated and scored," Nucleic Acids Research, vol. 39, no. 1, pp. D561-D568, 2011.

[66] B. Demchak, T. Hull, M. Reich et al., "Cytoscape: The network visualization tool for GenomeSpace workflows," F1000Research, vol. 3, article 151, 2014.

[67] P. V. Missiuro, K. Liu, L. Zou et al., "Information flow analysis of interactome networks," PLoS Computational Biology, vol. 5, no. 4, 2009.

[68] K. Raman, N. Damaraju, and G. K. Joshi, "The organisational structure of protein networks: Revisiting the centrality-lethality hypothesis," Systems and Synthetic Biology, vol. 8, no. 1, pp. 7381, 2014.

[69] Y. Zhang, M. Bai, B. Zhang et al., "Uncovering pharmacological mechanisms of Wu-tou decoction acting on rheumatoid arthritis through systems approaches: drug-target prediction, network analysis and experimental validation," Scientific Reports, vol. 5, article 9463, 2015.

[70] D. W. Huang, B. T. Sherman, Q. Tan et al., "The DAVID gene functional classification tool: a novel biological module-centric algorithm to functionally analyze large gene lists," Genome Biology, vol. 8, no. 9, article R183, 2007.

[71] M. E. Cooper, "Interaction of metabolic and haemodynamic factors in mediating experimental diabetic nephropathy," Diabetologia, vol. 44, no. 11, pp. 1957-1972, 2001.

[72] Y. S. Kanwar, L. Sun, P. Xie, F. Liu, and S. Chen, "A glimpse of various pathogenetic mechanisms of diabetic nephropathy," Annual Review of Pathology: Mechanisms of Disease, vol. 6, pp. 395-423, 2011.

[73] D. Fliser, K.-K. Wagner, A. Loos, D. Tsikas, and H. Haller, "Chronic angiotensin II receptor blockade reduces (intra)renal vascular resistance in patients with type 2 diabetes," Journal of the American Society of Nephrology, vol. 16, no. 4, pp. 1135-1140, 2005.

[74] F. Giacco and M. Brownlee, "Oxidative stress and diabetic complications," Circulation Research, vol. 107, no. 9, pp. 10581070, 2010.

[75] S. Fakhruddin, W. Alanazi, and K. E. Jackson, "DiabetesInduced Reactive Oxygen Species: Mechanism of Their Generation and Role in Renal Injury," Journal of Diabetes Research, vol. 2017, Article ID 8379327, 2017.

[76] M. Kamiyama, M. Urushihara, T. Morikawa et al., "Oxidative stress/angiotensinogen/renin-angiotensin system axis in patients with diabetic nephropathy," International Journal of Molecular Sciences, vol. 14, no. 11, pp. 23045-23062, 2013.

[77] H. Tomita, R. B. Sanford, O. Smithies, and M. Kakoki, "The kallikrein-kinin system in diabetic nephropathy," Kidney International, vol. 81, no. 8, pp. 733-744, 2012.

[78] V. Jakuš and N. Rietbrock, "Advanced glycation end-products and the progress of diabetic vascular complications," Physiological Research, vol. 53, no. 2, pp. 131-142, 2004.

[79] V. P. Singh, A. Bali, N. Singh, and A. S. Jaggi, "Advanced glycation end products and diabetic complications," Korean Journal of Physiology \& Pharmacology, vol. 18, no. 1, pp. 1-14, 2014.

[80] C. Hu, L. Sun, L. Xiao et al., "Insights into the mechanisms involved in the expression and regulation of extracellular matrix proteins in diabetic nephropathy," Current Medicinal Chemistry, vol. 22, no. 24, pp. 2858-2870, 2015.

[81] Y.-L. Yuan, C.-R. Guo, L.-L. Cui et al., “Timosaponin B-II ameliorates diabetic nephropathy via TXNIP, mTOR, and NF$\kappa \mathrm{B}$ signaling pathways in alloxan-induced mice," Drug Design, Development and Therapy, vol. 9, pp. 6247-6258, 2015.

[82] K. Sharma and F. N. Ziyadeh, "Hyperglycemia and diabetic kidney disease: the case for transforming growth factor- $\beta$ as a key mediator," Diabetes, vol. 44, no. 10, pp. 1139-1146, 1995.

[83] N. H. Kim, K. B. Kim, D. L. Kim et al., "Plasma and urinary vascular endothelial growth factor and diabetic nephropathy in type 2 diabetes mellitus," Diabetic Medicine, vol. 21, no. 6, pp. 545-551, 2004.

[84] K. Kanasaki, G. Taduri, and D. Koya, "Diabetic nephropathy: the role of inflammation in fibroblast activation and kidney fibrosis," Frontiers in Endocrinology, vol. 4, article 7, 2013.

[85] A. Diez-Sampedro, O. Lenz, and A. Fornoni, "Podocytopathy in diabetes: a metabolic and endocrine disorder," American Journal of Kidney Diseases, vol. 58, no. 4, pp. 637-646, 2011.

[86] C. Maric, C. Forsblom, L. Thorn, J. Wadén, and P.-H. Groop, "Association between testosterone, estradiol and sex hormone binding globulin levels in men with type 1 diabetes with nephropathy," Steroids, vol. 75, no. 11, pp. 772-778, 2010.

[87] N. González, I. Prieto, L. del Puerto-Nevado et al., "2017 update on the relationship between diabetes and colorectal cancer: epidemiology, potential molecular mechanisms and therapeutic implications," Oncotarget, vol. 8, no. 11, pp. 18456-18485, 2017.

[88] P. Xie, L. Sun, P. J. Oates, S. K. Srivastava, and Y. S. Kanwar, "Pathobiology of renal-specific oxidoreductase/myo-inositol oxygenase in diabetic nephropathy: Its implications in tubulointerstitial fibrosis," American Journal of Physiology-Renal Physiology, vol. 298, no. 6, pp. F1393-F1404, 2010.

[89] W.-N. Wang, W.-L. Zhang, G.-Y. Zhou et al., "Prediction of the molecular mechanisms and potential therapeutic targets for diabetic nephropathy by bioinformatics methods," International Journal of Molecular Medicine, vol. 37, no. 5, pp. 1181-1188, 2016. 
[90] J. L. Garvin, M. Herrera, and P. A. Ortiz, "Regulation of renal $\mathrm{NaCl}$ transport by nitric oxide, endothelin, and ATP: Clinical implications," Annual Review of Physiology, vol. 73, pp. 359-376, 2011.

[91] P. F. Mount and D. A. Power, "Nitric oxide in the kidney: Functions and regulation of synthesis," Acta Physiologica, vol. 187, no. 4, pp. 433-446, 2006.

[92] H. Kamijo, M. Higuchi, and K. Hora, "Chronic inhibition of nitric oxide production aggravates diabetic nephropathy in Otsuka Long-Evans Tokushima Fatty rats," Nephron Physiology, vol. 104, no. 1, pp. p12-p22, 2006.

[93] D. Ito, P. Cao, T. Kakihana et al., "Chronic running exercise alleviates early progression of nephropathy with upregulation of nitric oxide synthases and suppression of glycation in zucker diabetic rats," PLoS ONE, vol. 10, no. 9, Article ID e0138037, 2015.

[94] J. L. Evans, I. D. Goldfine, B. A. Maddux, and G. M. Grodsky, "Oxidative stress and stress-activated signaling pathways: a unifying hypothesis of type 2 diabetes," Endocrine Reviews, vol. 23, no. 5, pp. 599-622, 2002.

[95] F. N. Ziyadeh, "Mediators of diabetic renal disease: the case for TGF- $\beta$ as the major mediator," Journal of the American Society of Nephrology, vol. 15, no. 1, pp. S55-S57, 2004.

[96] M. Murphy, C. Godson, S. Cannon et al., "Suppression subtractive hybridization identifies high glucose levels as a stimulus for expression of connective tissue growth factor and other genes in human mesangial cells," The Journal of Biological Chemistry, vol. 274, no. 9, pp. 5830-5834, 1999.

[97] G. A. Sivaskandarajah, M. Jeansson, Y. Maezawa, V. Eremina, H. J. Baelde, and S. E. Quaggin, "Vegfa protects the glomerular microvasculature in diabetes," Diabetes, vol. 61, no. 11, pp. 29582966, 2012.

[98] S. Oltean, Y. Qiu, J. K. Ferguson et al., "Vascular endothelial growth factor- $\mathrm{A}_{165} \mathrm{~b}$ is protective and restores endothelial glycocalyx in diabetic nephropathy," Journal of the American Society of Nephrology, vol. 26, no. 8, pp. 1889-1904, 2015.

[99] Z. Dong and C. X. Chen, "Effect of catalpol on diabetic nephropathy in rats," Phytomedicine, vol. 20, no. 11, pp. 10231029, 2013.

[100] G.-d. Sun, C.-y. Li, W.-p. Cui et al., "Review of herbal traditional chinese medicine for the treatment of diabetic nephropathy," Journal of Diabetes Research, vol. 2016, Article ID 5749857, 18 pages, 2016.

[101] Y. Li, M. Zhao, D. He et al., "HDL in diabetic nephropathy has less effect in endothelial repairing than diabetes without complications," Lipids in Health and Disease, vol. 15, no. 1, article 76, 2016.

[102] H. Wu, L. Kong, S. Zhou et al., "The role of MicroRNAs in diabetic nephropathy," Journal of Diabetes Research, vol. 2014, Article ID 920134, 12 pages, 2014.

[103] E. Alonso-Merino, R. Martín Orozco, and L. Ruíz-Llorente, "Thyroid hormones inhibit TGF- $\beta$ signaling and attenuate fibrotic responses," Proceedings of the National Acadamy of Sciences of the United States of America, vol. 113, no. 24, pp. E3451-E3460, 2016.

[104] M. J. Rane, Y. Song, S. Jin et al., "Interplay between Akt and p38 MAPK pathways in the regulation of renal tubular cell apoptosis associated with diabetic nephropathy," American Journal of Physiology-Renal Physiology, vol. 298, no. 1, pp. F49-F61, 2010.

[105] H. Chen, S. Brahmbhatt, A. Gupta, and A. C. Sharma, "Duration of streptozotocin-induced diabetes differentially affects p38mitogen-activated protein kinase (MAPK) phosphorylation in renal and vascular dysfunction," Cardiovascular Diabetology, vol. 4, article 3, 2005. 


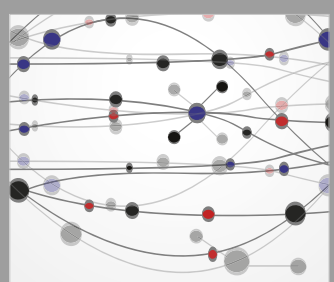

The Scientific World Journal
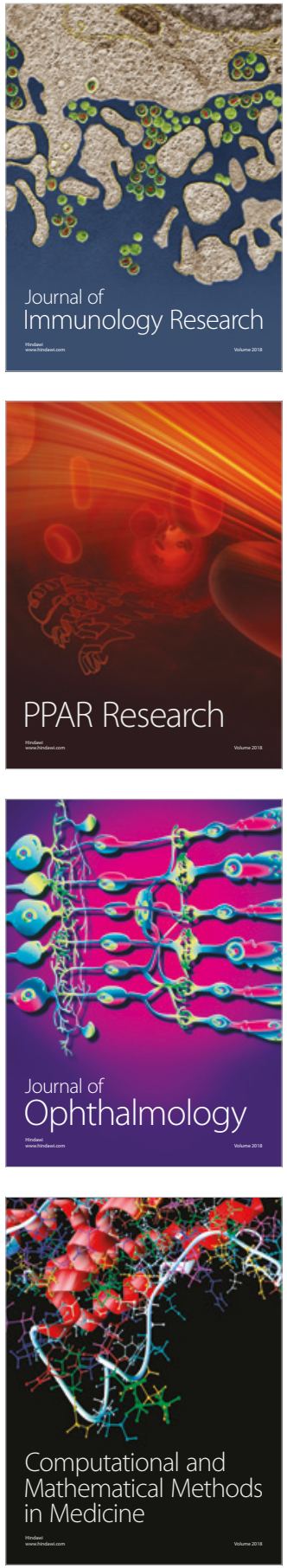

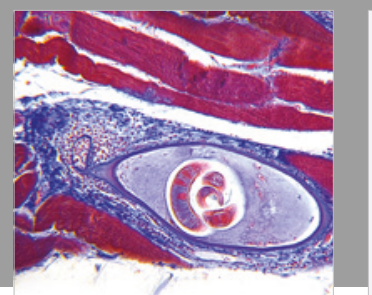

Gastroenterology Research and Practice

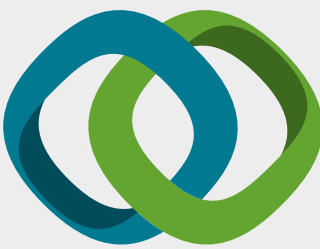

\section{Hindawi}

Submit your manuscripts at

www.hindawi.com
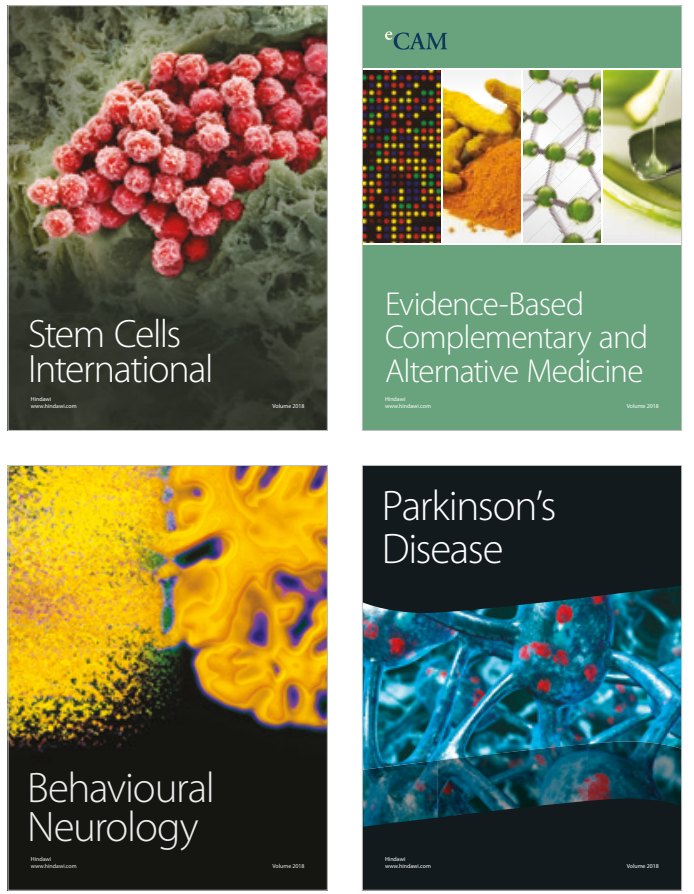

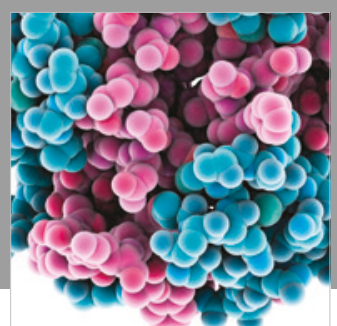

ournal of

Diabetes Research

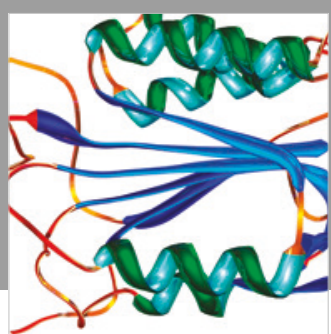

Disease Markers
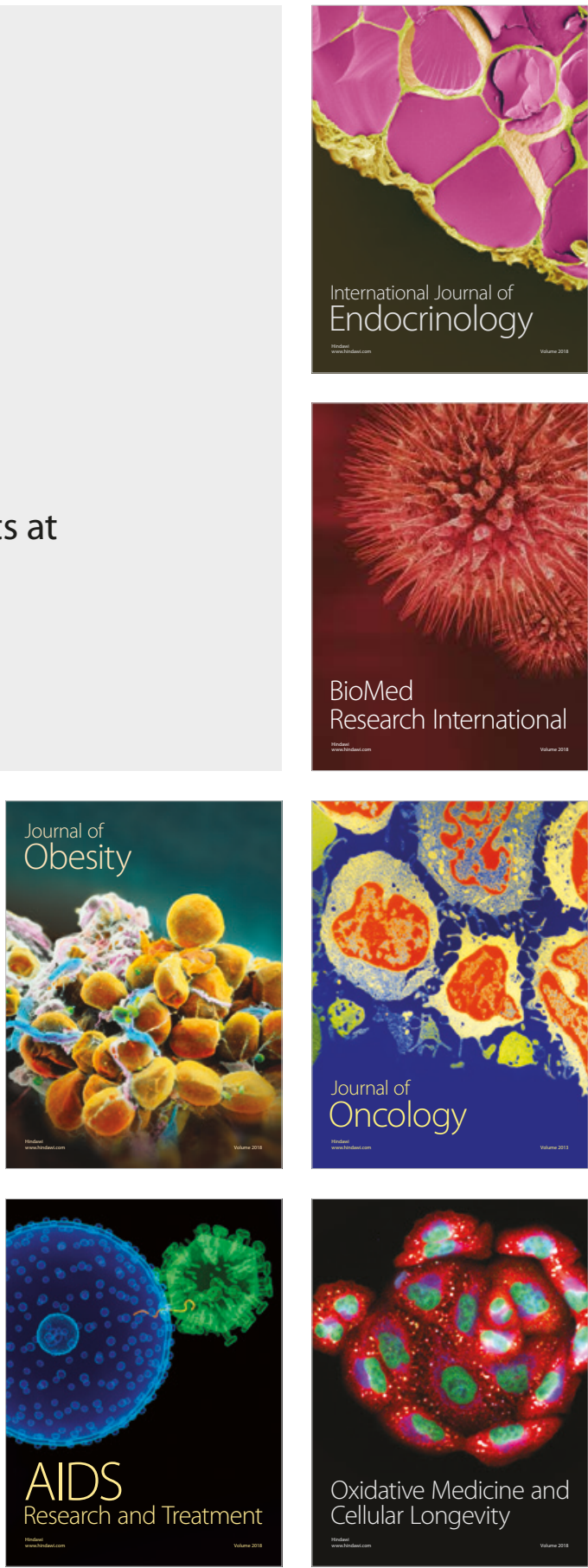